\title{
Homotopy Analysis Method for Three Types of Fractional Partial Differential Equations
}

\author{
Haidong Qu $\mathbb{D},{ }^{1}$ Zihang She, ${ }^{1,2}$ and Xuan Liu $\mathbb{D}^{1}$ \\ ${ }^{1}$ Department of Mathematics, Hanshan Normal University, Chaozhou 515041, China \\ ${ }^{2}$ Department of Mathematics, Shantou University, Shantou 515063, China \\ Correspondence should be addressed to Xuan Liu; czliuxuan@126.com
}

Received 22 January 2020; Accepted 1 June 2020; Published 10 July 2020

Academic Editor: Dimitri Volchenkov

Copyright (c) 2020 Haidong Qu et al. This is an open access article distributed under the Creative Commons Attribution License, which permits unrestricted use, distribution, and reproduction in any medium, provided the original work is properly cited.

In this paper, three types of fractional order partial differential equations, including the fractional Cauchy-Riemann equation, fractional acoustic wave equation, and two-dimensional space partial differential equation with time-fractional-order, are considered, and these models are obtained from the standard equations by replacing an integer-order derivative with a fractionalorder derivative in Caputo sense. Firstly, we discuss the fractional integral and differential properties of several functions which are derived from the Mittag-Leffler function. Secondly, by using the homotopy analysis method, the exact solutions for fractional order models mentioned above with suitable initial boundary conditions are obtained. Finally, we draw the computer graphics of the exact solutions, the approximate solutions (truncation of finite terms), and absolute errors in the limited area, which show that the effectiveness of the homotopy analysis method for solving fractional order partial differential equations.

\section{Introduction}

The concept of fractional derivatives can be traced back to a question raised by Marquis de L'Hopital to Gottfried Wilhelm Leibniz three hundred years ([1-3]) ago. Due to the lack of geometric or physical background support, fractional calculus has not entered the field of vision of most researchers. Until recent decades, some researchers have found that the fractional models are better than integer models in describing the chemical process, diffusion reaction, financial vibration, and other fields. Therefore, the fractional order problems gradually attracted the interest of many researchers, and the application expanded to many scientific fields including fluid flow, rheology, dynamical processes in self-similar and porous structures, diffusive transport akin to diffusion, electrical networks, probability and statistics, control theory of dynamical systems, viscoelasticity, electrochemistry of corrosion, optics and signal processing [4-8], and so on. These applications in interdisciplinary sciences motivate us to try to find out numerical or analytic solutions for the fractional differential equations.

Now, many effective methods for fractional differential equations have been presented, such as the finite difference method [9], spectral method [5], matrix approach [10], homotopy analysis method (HAM) [11], and homotopy perturbation method [12]. Especially, the HAM was first proposed by Liao in [13]. This method has been successfully applied to solve various linear or nonlinear problems $[8,14-18]$. The following is a brief survey.

In 2007, firstly, the HAM that was developed for an integer-order differential equation was directly extended to derive explicit and numerical solutions of nonlinear fractional differential equations by by Song and Zhang [19]. In 2008, Xu and Cang [20] employed the HAM to derive the solutions of the time fractional wave-like differential equations with a variable coefficient. In [21], HAM is applied to solve linear and nonlinear fractional initial-value problems by Hashim ea allenleadertwodots. In 2010, Dehghan et al. [22] applied HAM to solve several fractional KDV equations and showed the high accuracy and efficiency of the proposed technique. In [23], Abbasbandy et al. used HAM to obtain approximate solutions of fractional integrodifferential equations and gave some examples to illustrate the high efficiency and precision of this method. Very recently, Morales-Delgado et al. [24] have presented an analysis based 
on a combination of the Laplace transform and homotopy methods in order to provide new analytical approximated solutions of the fractional partial differential equations in the Liouville-Caputo and Caputo-Fabrizio senses. In 2019, an optimal homotopy analysis approach [25] was proposed to deal with nonlinear fractional differential equations, and the corresponding optimal initial approximation was discussed. For further information about the HAM, refer to [26-28].

In this paper, we use the HAM to solve fractional Cauchy-Riemann equations [29] and fractional acoustic wave equations [30], which are given by

$$
\begin{gathered}
\left\{\begin{array}{l}
{ }^{c} D_{0, t}^{\alpha} v(x, t)+{ }^{c} D_{0, x}^{\beta} \rho(x, t)=0, \\
{ }^{c} D_{0, t}^{\alpha} \rho(x, t)-{ }^{c} D_{0, x}^{\beta} v(x, t)=0,
\end{array}\right. \\
\left\{\begin{array}{l}
{ }^{c} D_{0, t}^{\alpha} v(x, t)+\frac{c_{0}^{2}}{b_{0}}{ }^{c} D_{0, x}^{\beta} \rho(x, t)=0, \\
{ }^{c} D_{0, t}^{\alpha} \rho(x, t)+b_{0}{ }^{c} D_{0, x}^{\beta} v(x, t)=0,
\end{array}\right.
\end{gathered}
$$

where $0<\alpha, \beta \leq 1$ and $b_{0}$ and $c_{0}$ are positive numbers and to solve the partial differential equation with a time-fractionalorder of the following form:

$$
{ }^{c} D_{0, t}^{\alpha} u(x, y, t)=\lambda \Delta u(x, y, t),
$$

with a time-fractional order $1<\alpha \leq 2$, and ${ }^{c} D_{0, t}^{\alpha}(\cdot)$ is the Caputo fractional derivative.

By setting $\alpha=1$ and $\beta=1$, the models (1) and (2) were transformed into Cauchy-Riemann equations and acoustic wave equations, respectively. Suppose that $v$ and $\rho$ satisfy the Cauchy-Riemann equations in an open subset of $R^{2}$, and consider the vector field $[v, \rho]^{T}$. In fluid dynamics, such a vector field is a potential flow. In the second model, $b_{0}$ and $c_{0}$ denote the medium density and the propagation velocity without an acoustic disturbance, respectively. Model (3) is a two-dimensional space and time-fractional-order model, which has important applications in many fields.

The rest of this paper is organized as follows. In Section 2 , the definitions and properties of fractional derivatives of some functions are introduced. Section 3 gives an introduction of the HAM which is used to solve fractional order differential equations. In Section 4, the analytic solutions for three types of fractional order differential equations were obtained by using the HAM, and the computer graphics of the exact solutions, the approximate solutions, and absolute errors were drawn in the limited area to clarify the effectiveness of the HAM. Finally, Section 5 offers some concluding remarks.

\section{Definitions and Lemmas}

Definition 1. (see [3]). A real function $f(x), x>0$, is said to be in the space $C_{\mu}, \mu \in R$, if there exists a real number $p>\mu$, such that $f(x)=x^{p} f_{1}(x)$, where $f_{1}(x) \in C_{(0, \infty)}$, and it is said to be in the space $C_{\mu}^{n}$, if and only if $f_{n} \in C_{\mu}, n \in N$.

Definition 2 (see [3]). The Riemann-Liouville fractional integral of order $\alpha \in R, \alpha>0$ of a function $f(x) \in C_{\mu}, \mu \geq-$ 1 is defined as

$$
\left(I_{0+}^{\alpha} f(t)\right)(x)=\frac{1}{\Gamma(\alpha)} \int_{0}^{x} \frac{f(t) \mathrm{d} t}{(x-t)^{1-\alpha}}, \quad x>0
$$

Definition 3 (see [3]). The Riemann-Liouville fractional derivative of order $\alpha \in R, \alpha>0$, on the usual Lebesgue space $L_{1}[a, b]$, is given by

$$
\begin{aligned}
\left(D_{0+}^{\alpha} f(t)\right)(x) & =\left(\frac{\mathrm{d}}{\mathrm{d} x}\right)^{n}\left(I_{0+}^{n-\alpha} f(t)\right)(x) \\
& =\frac{1}{\Gamma(n-\alpha)}\left(\frac{\mathrm{d}}{\mathrm{d} x}\right)^{n} \int_{0}^{x} \frac{f(t) \mathrm{d} t}{(x-t)^{\alpha-n+1}}
\end{aligned}
$$

where $(n=[\alpha]+1, x>0)$.

Definition 4 (see [3]). The Caputo fractional derivative of $f(x) \in C_{\mu}^{-1}, n \in N$, is defined as

$$
{ }^{c} D_{0, x}^{\alpha} f(x)= \begin{cases}I_{0+}^{n-\alpha}\left(\frac{\mathrm{d}}{\mathrm{d} x}\right)^{n} f(x), & n-1<\alpha<n, \\ \left(\frac{\mathrm{d}}{\mathrm{d} x}\right)^{n} f(x), & \alpha=n .\end{cases}
$$

Definition 5 (see [3]). The classical Mittag-Leffler function is defined by

$$
E_{\alpha}(Z):=\sum_{k=0}^{\infty} \frac{Z^{k}}{\Gamma(\alpha k+1)}, \quad Z \in C, \alpha>0 .
$$

The generalized Mittag-Leffler function is defined by

$$
E_{\alpha, \beta}(Z):=\sum_{k=0}^{\infty} \frac{Z^{k}}{\Gamma(\alpha k+\beta)}, \quad Z, \beta \in C, \alpha>0 .
$$

Definition 6 (see [3]). The functions $\sin _{\alpha}(Z), \cos _{\alpha}(Z)$, $\sin _{\alpha, \beta}(Z)$, and $\cos _{\alpha, \beta}(Z)(Z, \beta \in C, \alpha>0)$ are defined by

$$
\begin{aligned}
\sin _{\alpha}(Z) & =\sum_{k=1}^{\infty}(-1)^{k+1} \frac{Z^{2 k-1}}{\Gamma(\alpha(2 k-1)+1)}, \\
\cos _{\alpha}(Z) & =\sum_{k=0}^{\infty}(-1)^{k} \frac{Z^{2 k}}{\Gamma(\alpha(2 k)+1)}, \\
\sin _{\alpha, \beta}(Z) & =\sum_{k=1}^{\infty}(-1)^{k+1} \frac{Z^{2 k-1}}{\Gamma(\alpha(2 k-1)+\beta)}, \\
\cos _{\alpha, \beta}(Z) & =\sum_{k=0}^{\infty}(-1)^{k} \frac{Z^{2 k}}{\Gamma(\alpha(2 k)+\beta)} .
\end{aligned}
$$

Obviously, Euler's equations have the following forms:

$$
\begin{aligned}
E_{\alpha}(i Z) & =\cos _{\alpha}(Z)+i \sin _{\alpha}(Z), \\
E_{\alpha, \beta}(i Z) & =\cos _{\alpha, \beta}(Z)+i \sin _{\alpha, \beta}(Z) .
\end{aligned}
$$

In particular, when $\alpha=1$, we have 


$$
\begin{aligned}
& \sin _{1}(Z)=\sin (Z), \\
& \cos _{1}(Z)=\cos (Z),
\end{aligned}
$$

and when $\beta=1$, we have

$$
\begin{aligned}
& \sin _{\alpha, 1}(Z)=\sin _{\alpha}(Z), \\
& \cos _{\alpha, 1}(Z)=\cos _{\alpha}(Z) .
\end{aligned}
$$

Definition 7 (see [3]). The functions $\sinh _{\alpha}(Z), \cosh _{\alpha}(Z)$, $\sinh _{\alpha, \beta}(Z)$, and $\cosh _{\alpha, \beta}(Z)(Z, \beta \in C, \alpha>0)$ are defined by

$$
\begin{aligned}
\sinh _{\alpha}(Z) & =\sum_{k=1}^{\infty} \frac{Z^{2 k-1}}{\Gamma(\alpha(2 k-1)+1)} \\
\cosh _{\alpha}(Z) & =\sum_{k=0}^{\infty} \frac{Z^{2 k}}{\Gamma(\alpha(2 k)+1)} \\
\sinh _{\alpha, \beta}(Z) & =\sum_{k=1}^{\infty} \frac{Z^{2 k-1}}{\Gamma(\alpha(2 k-1)+\beta)}, \\
\cosh _{\alpha, \beta}(Z) & =\sum_{k=0}^{\infty} \frac{Z^{2 k}}{\Gamma(\alpha(2 k)+\beta)} .
\end{aligned}
$$

Obviously, the hyperbolic sine's and cosine's equations have the following forms:

$$
\begin{aligned}
\sinh _{\alpha}(Z) & =\frac{E_{\alpha}(Z)-E_{\alpha}(-Z)}{2}, \\
\cosh _{\alpha}(Z) & =\frac{E_{\alpha}(Z)+E_{\alpha}(-Z)}{2}, \\
\sinh _{\alpha, \beta}(Z) & =\frac{E_{\alpha, \beta}(Z)-E_{\alpha, \beta}(-Z)}{2}, \\
\cosh _{\alpha, \beta}(Z) & =\frac{E_{\alpha, \beta}(Z)+E_{\alpha, \beta}(-Z)}{2} .
\end{aligned}
$$

In particular, when $\alpha=1$, we have

$$
\begin{aligned}
& \sinh _{1}(Z)=\sinh (Z), \\
& \cosh _{1}(Z)=\cosh (Z) .
\end{aligned}
$$

When $\beta=1$, we have

$$
\begin{aligned}
& \sinh _{\alpha, 1}(Z)=\sinh _{\alpha}(Z), \\
& \cosh _{\alpha, 1}(Z)=\cosh _{\alpha}(Z) .
\end{aligned}
$$

Lemma 1. If $\sin _{\alpha}(Z), \cos _{\alpha}(Z), \sin _{\alpha, \beta}(Z)$, and $\cos _{\alpha, \beta}(Z)$ are defined as in Definition 6, then

$$
\begin{array}{r}
\left(I_{a+}^{\alpha}(t-a)^{\beta-1} \sin _{\mu, \beta}\left[\lambda(t-a)^{\mu}\right]\right)(x) \\
=(x-a)^{\alpha+\beta-1} \sin _{\mu, \alpha+\beta}\left[\lambda(t-a)^{\mu}\right], \\
\left(I_{a+}^{\alpha}(t-a)^{\beta-1} \cos _{\mu, \beta}\left[\lambda(t-a)^{\mu}\right]\right)(x) \\
=(x-a)^{\alpha+\beta-1} \cos _{\mu, \alpha+\beta}\left[\lambda(t-a)^{\mu}\right], \\
\left(D_{a+}^{\alpha}(t-a)^{\beta-1} \sin _{\mu, \beta}\left[\lambda(t-a)^{\mu}\right]\right)(x) \\
=(x-a)^{\beta-\alpha-1} \sin _{\mu, \beta-\alpha}\left[\lambda(t-a)^{\mu}\right], \\
\left(D_{a+}^{\alpha}(t-a)^{\beta-1} \cos _{\mu, \beta}\left[\lambda(t-a)^{\mu}\right]\right)(x) \\
=(x-a)^{\beta-\alpha-1} \cos _{\mu, \beta-\alpha}\left[\lambda(t-a)^{\mu}\right] .
\end{array}
$$

Proof. We denote the beta function by $\widetilde{\beta}$, and then, according to the definition of the Riemann-Liouville fractional integral, we have

$$
\begin{aligned}
& \left(I_{a+}^{\alpha}(t-a)^{\beta-1} \sin _{\mu, \beta}\left[\lambda(t-a)^{\mu}\right]\right)(x) \\
& \quad=\frac{1}{\Gamma(\alpha)} \int_{a+}^{x} \frac{(t-a)^{\beta-1} \sin _{\mu, \beta}\left[\lambda(t-a)^{\mu}\right]}{(x-t)^{1-\alpha}} \mathrm{d} t, \\
& \quad=\frac{1}{\Gamma(\alpha)} \int_{0+}^{x-a} \frac{\xi^{\beta-1} \sin _{\mu, \beta}\left[\lambda \xi^{\mu}\right]}{(x-\xi-a)^{1-\alpha}} \mathrm{d} \xi, \quad \xi=t-a, \\
& \quad=\frac{1}{\Gamma(\alpha)} \int_{0+}^{1} \frac{t^{\beta-1}(x-a)^{\beta-1} \sin _{\mu, \beta}\left[\lambda t^{\mu}(x-a)^{\mu}\right]}{(x-a)^{1-\alpha}(1-t)^{1-\alpha}} \cdot(x-a) \mathrm{d} t, \quad \xi=t(x-a), \\
& \quad=(x-a)^{\alpha+\beta-1} \frac{1}{\Gamma(\alpha)} \int_{0+}^{1} \frac{t^{\beta-1}}{(1-t)^{1-\alpha}} \cdot \sum_{k=1}^{\infty} \frac{(-1)^{k+1}\left[\lambda t^{\mu}(x-a)^{\mu}\right]^{2 k-1}}{\Gamma(\mu(2 k-1)+\beta)} \mathrm{d} t,
\end{aligned}
$$




$$
\begin{aligned}
& =(x-a)^{\alpha+\beta-1} \frac{1}{\Gamma(\alpha)} \sum_{k=1}^{\infty}(-1)^{k+1}\left[\lambda(x-a)^{\mu}\right]^{2 k-1} \cdot \int_{0+}^{1} \frac{t^{\beta-1} t^{\mu(2 k-1)}}{(1-t)^{1-\alpha} \Gamma(\mu(2 k-1)+\beta)} \mathrm{d} t \\
& =(x-a)^{\alpha+\beta-1} \frac{1}{\Gamma(\alpha)} \sum_{k}^{\infty} \frac{(-1)^{k+1}\left[\lambda(x-a)^{\mu}\right]^{2 k-1}}{\Gamma(\mu((2 k-1)+\beta)} \cdot \tilde{\beta}(\alpha, \mu(2 k-1)+\beta), \\
& =(x-a)^{\alpha+\beta-1} \frac{1}{\Gamma(\alpha)} \sum_{k=1}^{\infty} \frac{(-1)^{k+1}\left[\lambda(x-a)^{\mu}\right]^{2 k-1}}{\Gamma(\mu(2 k-1)+\beta)} \cdot \frac{\Gamma(\alpha) \Gamma(\mu(2 k-1)+\beta)}{\Gamma(\mu(2 k-1)+\beta+\alpha)} \\
& =(x-a)^{\alpha+\beta-1} \sum_{k=1}^{\infty} \frac{(-1)^{k+1}\left[\lambda(x-a)^{\mu}\right]^{2 k-1}}{\Gamma(\mu(2 k-1)+\beta+\alpha)} \\
& =(x-a)^{\alpha+\beta-1} \sin _{\mu, \alpha+\beta}\left[\lambda(t-a)^{\mu}\right] .
\end{aligned}
$$

Similarly, we obtain equation (18). Next, we will prove equation (19). From the definition of the Riemann-Liouville fractional derivative, we get

$$
\begin{aligned}
& \left(D_{a+}^{\alpha}(t-a)^{\beta-1} \sin _{\mu, \beta}\left[\lambda(t-a)^{\mu}\right]\right)(x) \\
& =\frac{1}{\Gamma(n-\alpha)}\left(\frac{\mathrm{d}}{\mathrm{d} x}\right)^{n} \int_{a+}^{x} \frac{(t-a)^{\beta-1} \sin _{\mu, \beta}\left[\lambda(t-a)^{\mu}\right]}{(x-t)^{\alpha-n+1}} \mathrm{~d} t \\
& =\frac{1}{\Gamma(n-\alpha)}\left(\frac{\mathrm{d}}{\mathrm{d} x}\right)^{n} \int_{0+}^{x-a} \frac{\xi^{\beta-1} \sin _{\mu, \beta}\left[\lambda \xi^{\mu}\right]}{(x-\xi-a)^{\alpha-n+1}} \mathrm{~d} \xi \\
& =\left(\frac{\mathrm{d}}{\mathrm{d} x}\right)^{n} \int_{0+}^{1} \frac{t^{\beta-1}(x-a)^{\beta-1} \sin _{\mu, \beta}\left[\lambda t^{\mu}(x-a)^{\mu}\right]}{(x-a)^{\alpha-n+1}(1-t)^{\alpha-n+1}} \cdot \frac{1}{\Gamma(n-\alpha)}(x-a) \mathrm{d} t \quad(\xi=t(x-a)) \\
& =\left(\frac{\mathrm{d}}{\mathrm{d} x}\right)^{n} \frac{(x-a)^{\beta-\alpha+n-1}}{\Gamma(n-\alpha)} \cdot \int_{0+}^{1} \frac{t^{\beta-1}}{(1-t)^{\alpha-n+1}} \sum_{k=1}^{\infty} \frac{(-1)^{k+1}\left[\lambda t^{\mu}(x-a)^{\mu}\right]^{2 k-1}}{\Gamma(\mu(2 k-1)+\beta)} \mathrm{d} t \\
& =\left(\frac{\mathrm{d}}{\mathrm{d} x}\right)^{n} \frac{(x-a)^{\beta-\alpha+n-1}}{\Gamma(n-\alpha)} \sum_{k=1}^{\infty} \frac{(-1)^{k+1}\left[\lambda(x-a)^{\mu}\right]^{2 k-1}}{\Gamma(\mu(2 k-1)+\beta)} \cdot \frac{\Gamma(n-\alpha) \Gamma(\mu(2 k-1)+\beta)}{\Gamma(\mu(2 k-1)+\beta-\alpha+n)} \\
& =(x-a)^{\beta-\alpha-1} \sum_{k=1}^{\infty} \frac{(-1)^{k+1}\left[\lambda(x-a)^{\mu}\right]^{2 k-1}}{\Gamma(\mu(2 k-1)+\beta-\alpha)} \\
& =(x-a)^{\beta-\alpha-1} \sin _{\mu, \beta-\alpha}\left[\lambda(t-a)^{\mu}\right] .
\end{aligned}
$$

Similarly, we obtain equation (20). In particular, when $\beta=1, \mu=\alpha$, we have

$$
\begin{aligned}
\left(D_{a+}^{\alpha} \sin _{\alpha}\left[\lambda(t-a)^{\alpha}\right]\right)(x) & =\lambda \cos _{\alpha}\left[\lambda(x-a)^{\alpha}\right], \\
\left(D_{a+}^{\alpha}\left\{\cos _{\alpha}\left[\lambda(t-a)^{\alpha}\right]-1\right\}\right)(x) & =-\lambda \sin _{\alpha}\left[\lambda(x-a)^{\alpha}\right] .
\end{aligned}
$$

While replacing the Riemann-Liouville fractional derivative with the Caputo derivative, we get the following forms:

$$
\begin{aligned}
& \left({ }^{c} D_{a+}^{\alpha} \sin _{\alpha}\left[\lambda(t-a)^{\alpha}\right]\right)(x)=\lambda \cos _{\alpha}\left[\lambda(x-a)^{\alpha}\right], \\
& \left({ }^{c} D_{a+}^{\alpha} \cos _{\alpha}\left[\lambda(t-a)^{\alpha}\right]\right)(x)=-\lambda \sin _{\alpha}\left[\lambda(x-a)^{\alpha}\right] .
\end{aligned}
$$


Lemma 2. If $\sinh _{\alpha}(Z), \quad \cosh _{\alpha}(Z), \quad \sinh _{\alpha, \beta}(Z), \quad$ and $\cosh _{\alpha, \beta}(Z)$ are defined as in Definition 7, then

$$
\begin{aligned}
& \left(I_{a+}^{\alpha}(t-a)^{\beta-1} \sinh _{\mu, \beta}\left[\lambda(t-a)^{\mu}\right]\right)(x)=(x-a)^{\alpha+\beta-1} \sinh _{\mu, \alpha+\beta}\left[\lambda(t-a)^{\mu}\right], \\
& \left(I_{a+}^{\alpha}(t-a)^{\beta-1} \cosh _{\mu, \beta}\left[\lambda(t-a)^{\mu}\right]\right)(x)=(x-a)^{\alpha+\beta-1} \cosh _{\mu, \alpha+\beta}\left[\lambda(t-a)^{\mu}\right], \\
& \left(D_{a+}^{\alpha}(t-a)^{\beta-1} \sinh _{\mu, \beta}\left[\lambda(t-a)^{\mu}\right]\right)(x)=(x-a)^{\beta-\alpha-1} \sinh _{\mu, \beta-\alpha}\left[\lambda(t-a)^{\mu}\right], \\
& \left(D_{a+}^{\alpha}(t-a)^{\beta-1} \cosh _{\mu, \beta}\left[\lambda(t-a)^{\mu}\right]\right)(x)=(x-a)^{\beta-\alpha-1} \cosh _{\mu, \beta-\alpha}\left[\lambda(t-a)^{\mu}\right] .
\end{aligned}
$$

Proof. Similar to the proof of Lemma 1, we obtain equations (25)-(28). In particular, when $\beta=1$ and $\mu=\alpha$,

$$
\begin{aligned}
\left(D_{a+}^{\alpha} \sinh _{\alpha}\left[\lambda(t-a)^{\alpha}\right]\right)(x) & =\lambda \cosh _{\alpha}\left[\lambda(x-a)^{\alpha}\right], \\
\left(D_{a+}^{\alpha}\left\{\cosh _{\alpha}\left[\lambda(t-a)^{\alpha}\right]-1\right\}\right)(x) & =\lambda \sinh _{\alpha}\left[\lambda(x-a)^{\alpha}\right] .
\end{aligned}
$$

While, replacing the Riemann-Liouville fractional derivative with the Caputo derivative, we get the following forms:

$$
\begin{aligned}
& \left({ }^{c} D_{a+}^{\alpha} \sinh _{\alpha}\left[\lambda(t-a)^{\alpha}\right]\right)(x)=\lambda \cosh _{\alpha}\left[\lambda(x-a)^{\alpha}\right], \\
& \left({ }^{c} D_{a+}^{\alpha} \cosh _{\alpha}\left[\lambda(t-a)^{\alpha}\right]\right)(x)=\lambda \sinh _{\alpha}\left[\lambda(x-a)^{\alpha}\right] .
\end{aligned}
$$

\section{HAM}

In this section, we consider a linear or nonlinear equation in a general form:

$$
N[u(x, t)]=0,
$$

where $u(x, t)$ is an unknown function and $x$ and $t$ are independent variables. Let $u_{0}(x, t)$ denote an initial approximation of the solution of equation (31), $h$ a nonzero auxiliary parameter, $H(x, t)$ a nonzero auxiliary function, and $L$ is an auxiliary linear operator. Then, we construct the HAM deformation equation in the following form:

$$
(1-q) L\left[\Phi(x, t ; q)-u_{0}(x, t)\right]=q h H(x, t) N[\Phi(x, t ; q)],
$$

where $q \in[0,1]$ is an embedding parameter. Obviously, when $q=0$ and $q=1$, the abovementioned HAM deformation equation (32) has the solutions

$$
\begin{aligned}
& \Phi(x, t ; 0)=u_{0}(x, t), \\
& \Phi(x, t ; 1)=u(x, t),
\end{aligned}
$$

respectively. Thus, as $q$ increases from 0 to $1, \Phi(x, t ; q)$ varies from the initial guesses $\Phi(x, t ; 0)$ to the solution $\Phi(x, t ; 1)$ of equation (31). Expanding $\Phi(x, t ; q)$ in Taylor's series with respect to $q$, we have

$$
\Phi(x, t ; q)=u_{0}(x, t)+\sum_{m=1}^{\infty} u_{m}(x, t) q^{m}
$$

where

$$
u_{m}(x, t)=\left.\frac{1}{m !} \frac{\partial^{m} \Phi(x, t ; q)}{\partial q^{m}}\right|_{q=0} .
$$

For brevity, we define a vector

$$
\overrightarrow{u_{m}}=\left\{u_{0}, u_{1}, \ldots, u_{m}\right\}
$$

Differentiating the HAM deformation equation (32) $m$ times with respect to $q$, then setting $q=0$, and finally dividing it by $m$ !, we obtain the $m$ th-order deformation equation:

$$
L\left[u_{m}-\chi_{m} u_{m-1}\right]=h H(x, t) R_{m}\left(\overrightarrow{u_{m-1}}\right),
$$

where

$$
\begin{gathered}
R_{m}\left(\overrightarrow{u_{m-1}}\right)=\left.(1 /(m-1) !)\left(\partial^{m-1} N[\Phi(x, t ; q)] / \partial q^{m-1}\right)\right|_{q=0} \text {, and } \\
\chi_{m}= \begin{cases}0, & m=1, \\
1, & m>1 .\end{cases}
\end{gathered}
$$

Operating the inverse operator of $L$ on both sides of equation (37), we have

$$
u_{m}(x, t)=\chi_{m} u_{m-1}(x, t)+h H(x, t) L^{-1} R_{m}\left(\overrightarrow{u_{m-1}(x, t)}\right) .
$$

In this way, it is easy to obtain $u_{1}(x, t), u_{2}(x, t), \ldots$ one after another, and then, we get an exact solution of the original equation (31) of the series form:

$$
u(x, t)=\sum_{m=0}^{\infty} u_{m}(x, t)
$$

\section{Applying HAM}

In this section, we present three examples to illustrate the applicability of HAM for solving FCPDEs introduced in Section 3. For more details about the HAM, the reader can refer to [8], [13-16].

4.1. The Fractional Cauchy-Riemann Equations. In this section, we consider the fractional Cauchy-Riemann equation (1) with the following initial conditions: 


$$
\begin{aligned}
& v(x, 0)=\sin _{\beta} x^{\beta}=v_{0}(x, t), \\
& \rho(x, 0)=\cos _{\beta} x^{\beta}=\rho_{0}(x, t) .
\end{aligned}
$$

First, we choose two linear fractional order operators:

$$
\begin{aligned}
& L_{1}[\Theta(x, t ; q)]={ }^{c} D_{0, t}^{\alpha} \Theta(x, t ; q), \\
& L_{2}[\Lambda(x, t ; q)]={ }^{c} D_{0, t}^{\alpha} \Lambda(x, t ; q) .
\end{aligned}
$$

Secondly, we define two linear operators as

$$
\begin{aligned}
& N_{1}[\Theta(x, t ; q), \Lambda(x, t ; q)]={ }^{c} D_{0, t}^{\alpha} \Theta(x, t ; q)+{ }^{c} D_{0, x}^{\beta} \Lambda(x, t ; q), \\
& N_{2}[\Lambda(x, t ; q), \Theta(x, t ; q)]={ }^{c} D_{0, t}^{\alpha} \Lambda(x, t ; q)-{ }^{c} D_{0, x}^{\beta} \Theta(x, t ; q) .
\end{aligned}
$$

Using the abovementioned definitions and with the assumption $H(x, t)=1$, we construct the zeroth-order deformation equations (ZDE):

$$
\begin{array}{r}
(1-q) L_{1}\left[\Theta(x, t ; q)-v_{0}(x, t)\right]=q h N_{1}[\Theta(x, t ; q), \Lambda(x, t ; q)], \\
(1-q) L_{2}\left[\Lambda(x, t ; q)-\rho_{0}(x, t)\right]=q h N_{2}[\Lambda(x, t ; q), \Theta(x, t ; q)] .
\end{array}
$$

Thirdly, differentiating the ZDEs $m$ times with respect to $q$, then setting $q=0$, and dividing it by $m$ !, we get the $m$ thorder deformation equations:

$$
\begin{aligned}
& L_{1}\left[v_{m}(x, t)-\chi_{m} v_{m-1}(x, t)\right] \\
& =h N_{1}\left[\overrightarrow{R_{m}}\left(v_{m-1}(x, t), \rho_{m-1}(x, t)\right)\right], \\
& L_{2}\left[\rho_{m}(x, t)-\chi_{m} \rho_{m-1}(x, t)\right] \\
& =h N_{2}\left[\overrightarrow{R_{m}}\left(\rho_{m-1}(x, t), v_{m-1}(x, t)\right)\right],
\end{aligned}
$$

where

$$
\begin{aligned}
\chi_{m}= & \begin{cases}0, & m=1, \\
1, & m>1,\end{cases} \\
& N_{1}\left[\overrightarrow{R_{m}}\left(v_{m-1}(x, t), \rho_{m-1}(x, t)\right)\right] \\
= & { }^{c} D_{0, t}^{\alpha} v_{m-1}(x, t ; q)+{ }^{c} D_{0, x}^{\beta} \rho_{m-1}(x, t ; q), \\
& N_{2}\left[\overrightarrow{R_{m}}\left(\rho_{m-1}(x, t), v_{m-1}(x, t)\right)\right] \\
= & { }^{c} D_{0, t}^{\alpha} \rho_{m-1}(x, t ; q)-{ }^{c} D_{0, x}^{\beta} v_{m-1}(x, t ; q) .
\end{aligned}
$$

Finally, operating the operators $L_{1}^{-1}$ and $L_{2}^{-1}$ on both the sides of equations (44) and (45), respectively, we have

$$
\begin{aligned}
v_{m}(x, t)= & \chi_{m} v_{m-1}(x, t) \\
& +h L_{1}^{-1} N_{1}\left[\overrightarrow{R_{m}}\left(\left(v_{m-1}(x, t), \rho_{m-1}(x, t)\right)\right],\right. \\
\rho_{m}(x, t)= & \chi_{m} \rho_{m-1}(x, t) \\
& +h L_{2}^{-1} N_{2}\left[\overrightarrow{R_{m}}\left(\rho_{m-1}(x, t), v_{m-1}(x, t)\right)\right] .
\end{aligned}
$$

$$
\begin{aligned}
& v_{0}(x, t)=\sin _{\beta} x^{\beta}, \\
& \rho_{0}(x, t)=\cos _{\beta} x^{\beta},
\end{aligned}
$$

$$
\begin{aligned}
v_{1}(x, t) & =0+h L_{1}^{-1} N_{1}\left[\overrightarrow{R_{1}}\left(v_{0}(x, t), \rho_{0}(x, t)\right)\right], \\
& =h L_{1}^{-1}\left({ }^{c} D_{0, t}^{\alpha} v_{0}(x, t ; q)+{ }^{c} D_{0, x}^{\beta} \rho_{0}(x, t ; q)\right), \\
& =h I_{0, t}^{\alpha}\left(0-\sin _{\beta} x^{\beta}\right), \\
& =-h \sin _{\beta} x^{\beta} \frac{t^{\alpha}}{\Gamma(1+\alpha)},
\end{aligned}
$$

$$
\begin{aligned}
\rho_{1}(x, t) & =0+h L_{2}^{-1} N_{1}\left[\overrightarrow{R_{1}}\left(\rho_{0}(x, t), v_{0}(x, t)\right)\right], \\
& =h L_{2}^{-1}\left({ }^{c} D_{0, t}^{\alpha} \rho_{0}(x, t)-{ }^{c} D_{0, x}^{\beta} v_{0}(x, t)\right), \\
& =h I_{0, t}^{\alpha}\left(0-\cos _{\beta} x^{\beta}\right), \\
& =-h \cos _{\beta} x^{\beta} \frac{t^{\alpha}}{\Gamma(1+\alpha)},
\end{aligned}
$$

$$
\begin{aligned}
v_{2}(x, t) & =v_{1}(x, t)+h L_{1}^{-1} N_{1}\left[\overrightarrow{R_{2}}\left(v_{1}(x, t), \rho_{1}(x, t)\right)\right], \\
& =v_{1}(x, t)+h L_{1}^{-1}\left({ }^{c} D_{0, t}^{\alpha} v_{1}(x, t)+{ }^{c} D_{0, x}^{\beta} \rho_{1}(x, t)\right), \\
& =v_{1}(x, t)+h L_{1}^{-1} L_{1} v_{1}(x, t)
\end{aligned}
$$$$
+h I_{0, t}^{\alpha}\left(-h \sin _{\beta} x^{\beta} \frac{t^{\alpha}}{\Gamma(1+\alpha)}\right),
$$$$
=-h \sin _{\beta} x^{\beta} \frac{t^{\alpha}}{\Gamma(1+\alpha)}
$$

$$
\begin{aligned}
& -h^{2} \sin _{\beta} x^{\beta} \frac{t^{\alpha}}{\Gamma(1+\alpha)}+h^{2} \sin _{\beta} x^{\beta} \frac{t^{2 \alpha}}{\Gamma(1+2 \alpha)}, \\
\rho_{2}(x, t)= & \rho_{1}(x, t)+h L_{2}^{-1} N_{2}\left[\overrightarrow{R_{2}}\left(\rho_{1}(x, t), v_{1}(x, t)\right)\right], \\
= & \rho_{1}(x, t)+h L_{2}^{-1}\left({ }^{c} D_{0, t}^{\alpha} \rho_{1}(x, t)-{ }^{c} D_{0, x}^{\beta} v_{1}(x, t)\right), \\
= & \rho_{1}(x, t)+h L_{2}^{-1} L_{2} \rho_{1}(x, t) \\
& +h I_{0, t}^{\alpha}\left(h \cos _{\beta} x^{\beta} \frac{t^{\alpha}}{\Gamma(1+\alpha)}\right), \\
= & -h \cos _{\beta} x^{\beta} \frac{t^{\alpha}}{\Gamma(1+\alpha)} \\
& -h^{2} \cos _{\beta} x^{\beta} \frac{t^{\alpha}}{\Gamma(1+\alpha)}+h^{2} \cos _{\beta} x^{\beta} \frac{t^{2 \alpha}}{\Gamma(1+2 \alpha)},
\end{aligned}
$$

From Lemma 1 and calculating one by one, we get 
By repeating this procedure for $h=-1$ and noting Definition 5, we obtain the exact solution:

$$
\begin{aligned}
v(x, t) & =\left.\sum_{k=0}^{\infty} v_{k}(x, t)\right|_{h=-1}, \\
& =\sin _{\beta} x^{\beta}\left(1+\frac{t^{\alpha}}{\Gamma(1+\alpha)}+\frac{t^{2 \alpha}}{\Gamma(1+2 \alpha)}+\cdots\right), \\
& =\sin _{\beta} x^{\beta} E_{\alpha}\left(t^{\alpha}\right), \\
\rho(x, t) & =\left.\sum_{k=0}^{\infty} \rho_{k}(x, t)\right|_{h=-1}, \\
& =\cos _{\beta} x^{\beta}\left(1+\frac{t^{\alpha}}{\Gamma(1+\alpha)}+\frac{t^{2 \alpha}}{\Gamma(1+2 \alpha)}+\cdots\right), \\
& =\cos _{\beta} x^{\beta} E_{\alpha}\left(t^{\alpha}\right) .
\end{aligned}
$$

More interestingly, when $\alpha=1$ and $\beta=1$,

$$
\begin{aligned}
& v(x, t)=\sin x e^{t}, \\
& \rho(x, t)=\cos x e^{t}
\end{aligned}
$$

are the exact solutions of Cauchy-Riemann equations

$$
\left\{\begin{array}{l}
v_{t}(x, t)+\rho_{x}(x, t)=0, \\
\rho_{t}(x, t)-v_{x}(x, t)=0,
\end{array}\right.
$$

with initial conditions $v(x, 0)=\sin x$ and $\rho(x, 0)=\cos x$. We draw the computer graphics of the exact solutions $v(x, t)$ and $\rho(x, t)$ by using MATLAB software; see Figures 1-4. Figures $5-8$ show the approximate solution obtained by the HAM with intercepting 15 items, and the absolute errors of exact solutions and approximate solutions are shown in Figures 9-12, with different values $\alpha$ and $\beta$. In the process of compiling the program, we calculate $\cos _{\alpha, \beta}(x)$ and $\sin _{\alpha, \beta}(x)$ by the formulas $\cos _{\alpha, \beta}(x)=(1 / 2)\left(E_{\alpha, \beta}(i x)+E_{\alpha, \beta}(-i x)\right)$ and $\sin _{\alpha, \beta}(x)=(1 / 2 i)\left(E_{\alpha, \beta}(i x)-E_{\alpha, \beta}(-i x)\right)$, respectively. The MATLAB code for the Mittag-Leffler function was provided by Podlubny [31].

4.2. Fractional Acoustic Wave Equations. In this section, we consider fractional acoustic wave equation (2) with initial conditions:

$$
\begin{aligned}
& v(x, 0)=E_{\beta}\left(x^{\beta}\right)=v_{0}(x, t), \\
& \rho(x, 0)=\sin _{\beta} x^{\beta}=\rho_{0}(x, t) .
\end{aligned}
$$

First, the choose two linear fractional order operators:

$$
\begin{aligned}
& L_{1}[\Theta(x, t ; q)]={ }^{c} D_{0, t}^{\alpha} \Theta(x, t ; q), \\
& L_{2}[\Lambda(x, t ; q)]={ }^{c} D_{0, t}^{\alpha} \Lambda(x, t ; q) .
\end{aligned}
$$

Secondly, we define two linear operators as

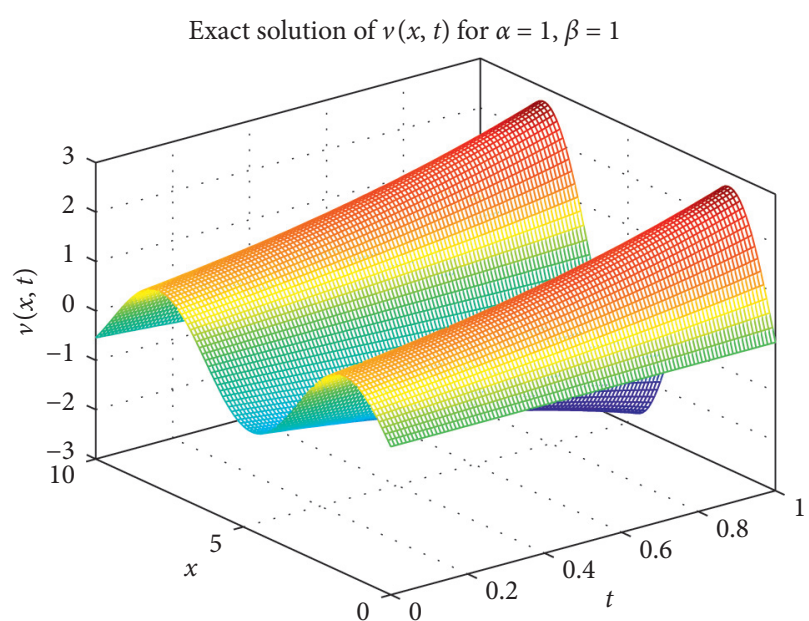

Figure 1: The exact solution $v(x, t)$ to model (1) with $\alpha=\beta=1$.

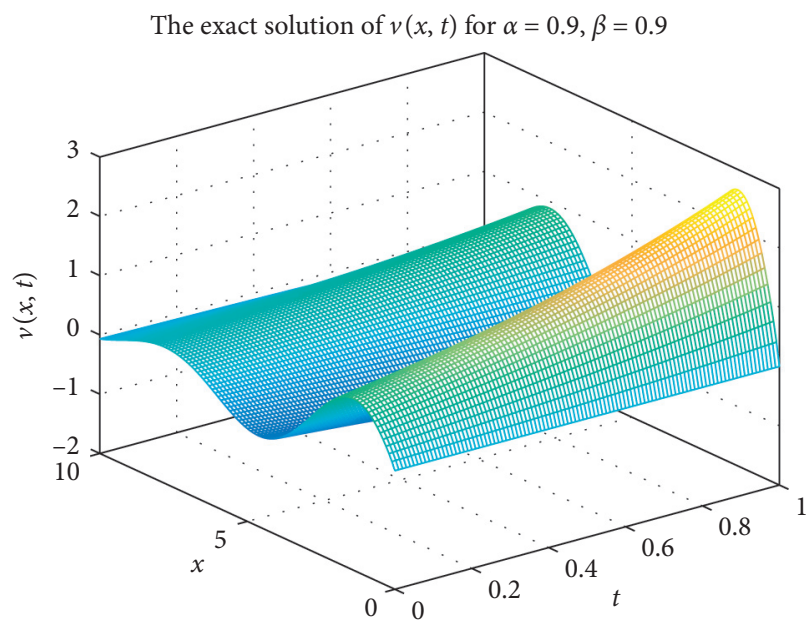

Figure 2: The exact solution $v(x, t)$ to model (1) with $\alpha=\beta=0.9$.

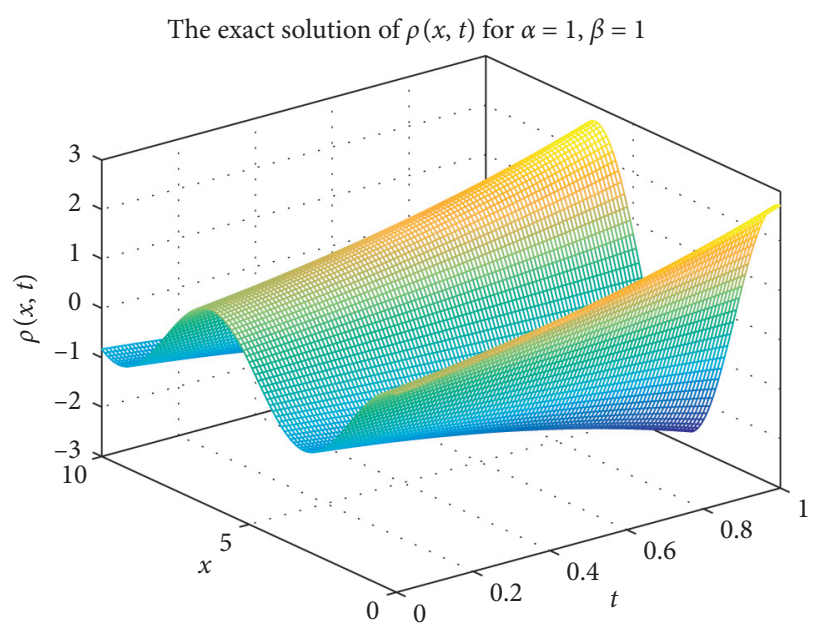

Figure 3: The exact solution $\rho(x, t)$ to model (1) with $\alpha=\beta=1$. 


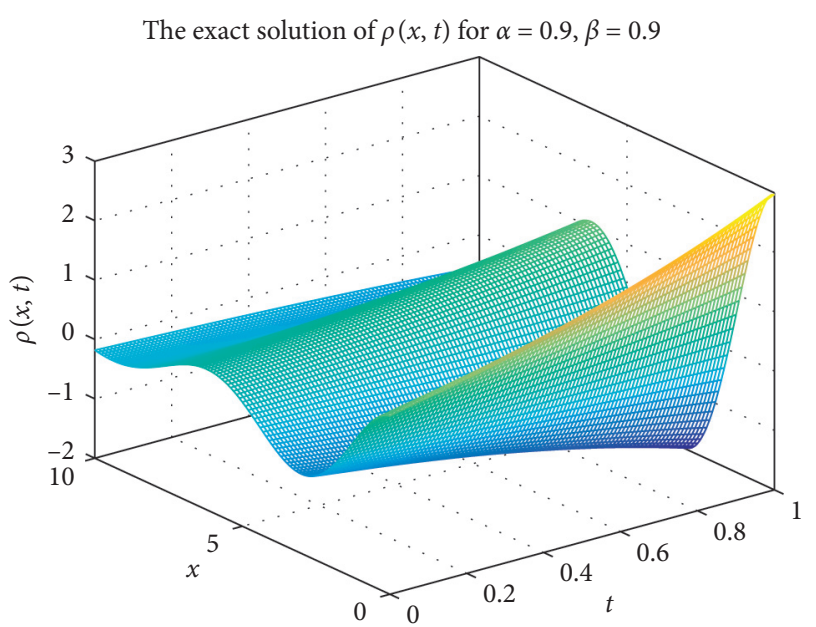

FIGURE 4: The exact solution $\rho(x, t)$ to model (1) with $\alpha=\beta=0.9$.

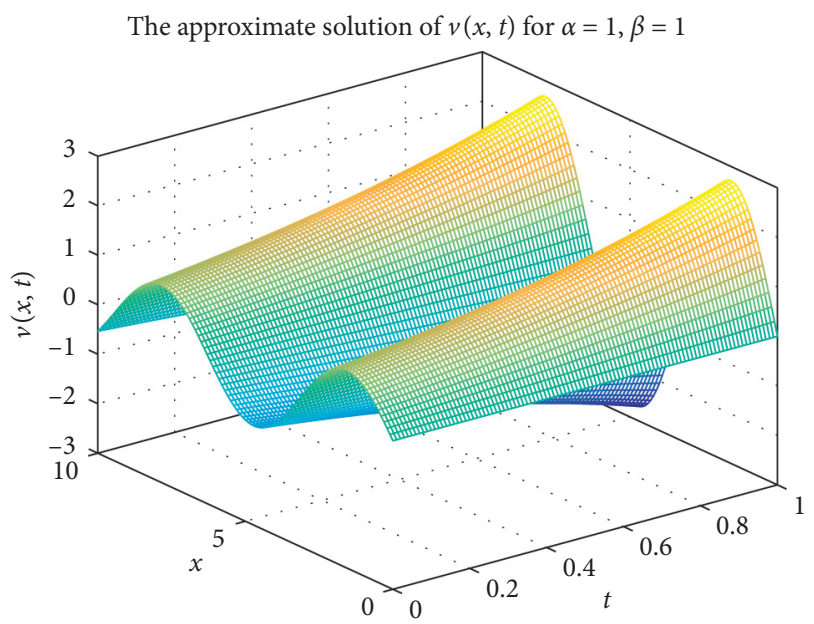

FIGURE 5: The approximate solution of $v(x, t)$ to model (1) with $\alpha=\beta=1$ (truncate 15 items that were obtained by the HAM).

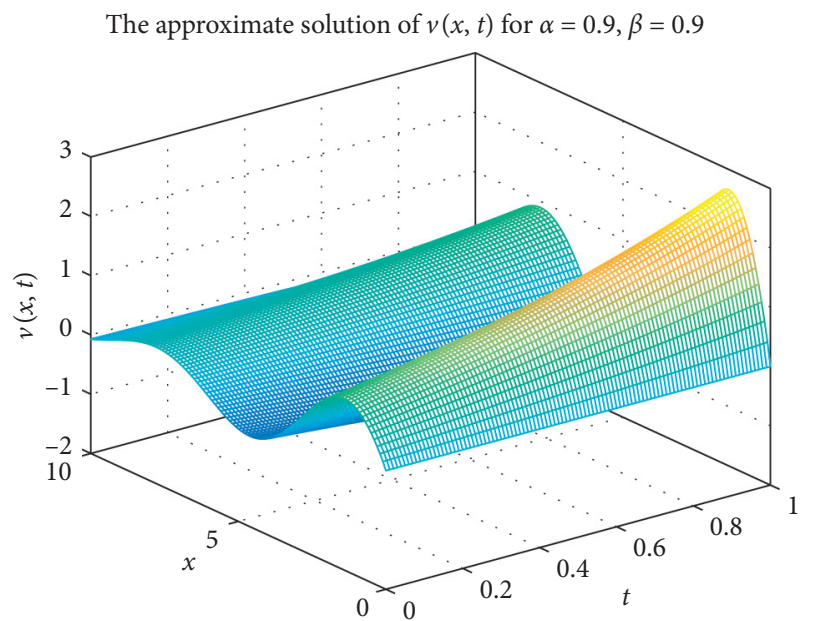

FIgURE 6: The approximate solution $v(x, t)$ to model (1) with $\alpha=$ $\beta=0.9$ (truncate 15 items that were obtained by the HAM).

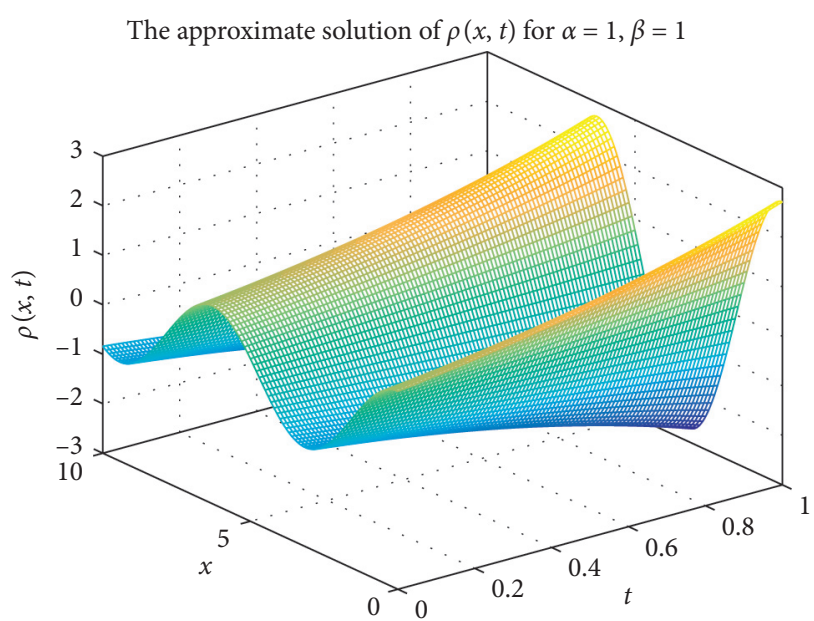

FIGURE 7: The approximate solution of $\rho(x, t)$ to model (1) with $\alpha=\beta=1$ (truncate 15 items that were obtained by the HAM).

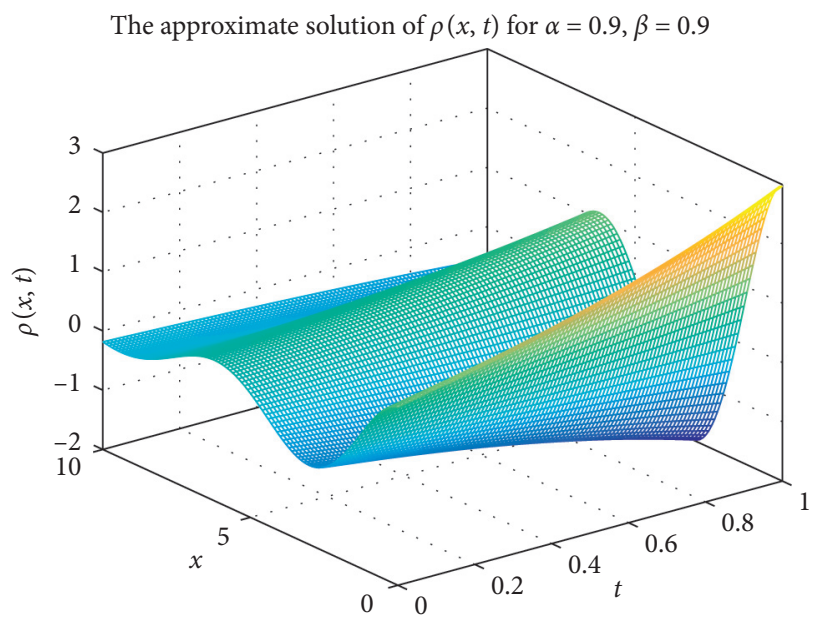

FIGURE 8: The approximate solution of $\rho(x, t)$ to model (1) with $\alpha=\beta=0.9$ (truncate 15 items that were obtained by the HAM).

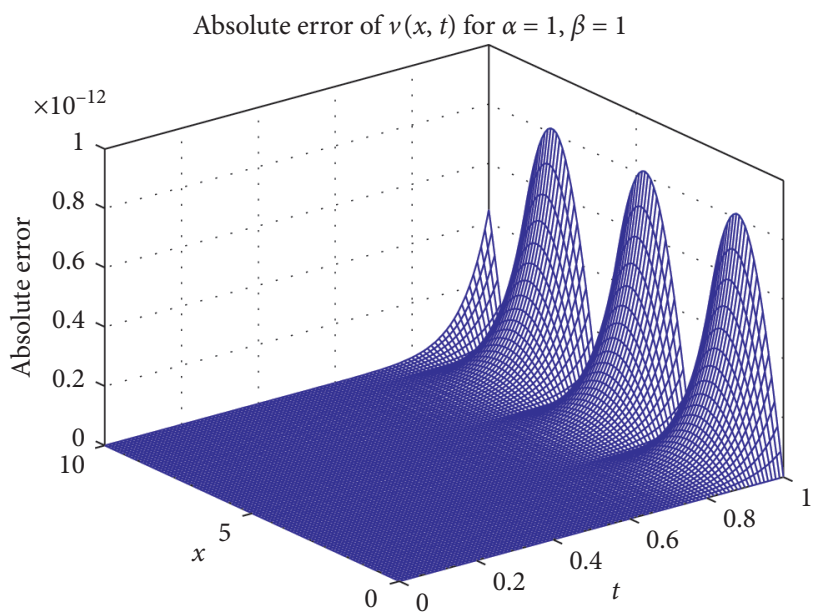

FIgURE 9: Absolute error of the exact solution and approximate solution $v(x, t)$ to model (1) with $\alpha=\beta=1$. 


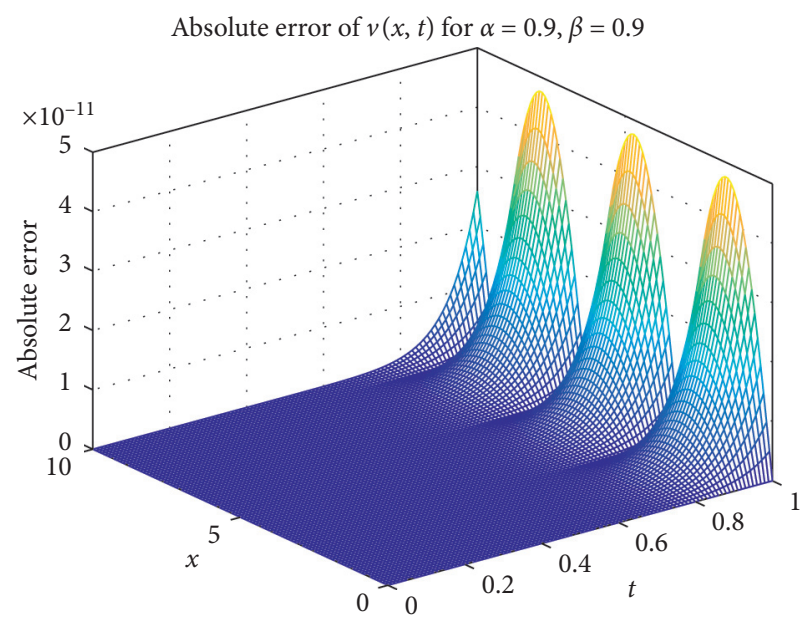

Figure 10: Absolute error of the exact solution and approximate solution $v(x, t)$ to model (1) with $\alpha=\beta=0.9$.

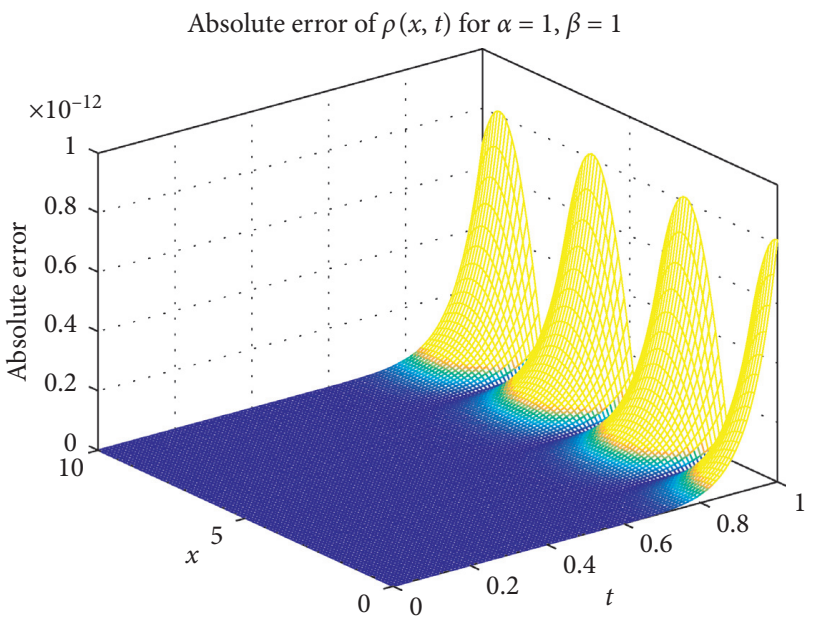

Figure 11: Absolute error of the exact solution and approximate solution $\rho(x, t)$ to model (1) with $\alpha=\beta=1$.

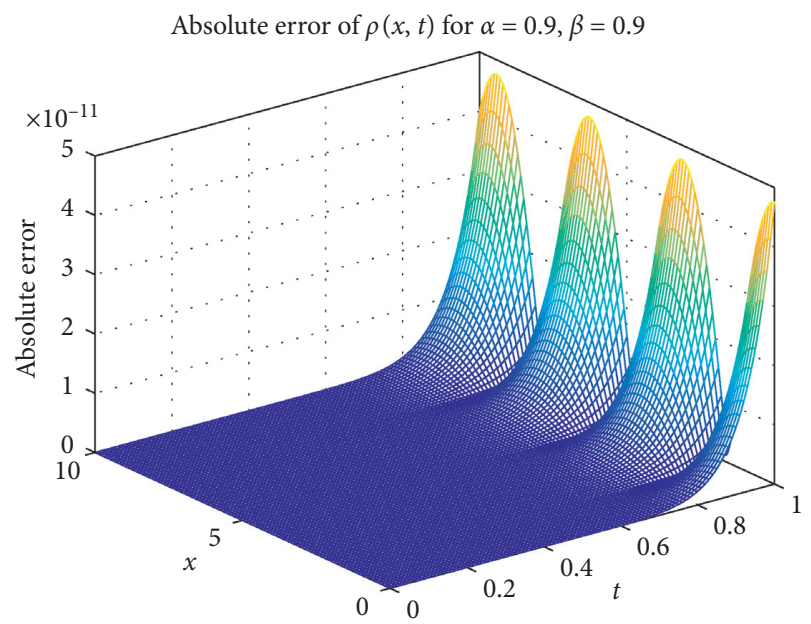

FIgURE 12: Absolute error of the exact solution and approximate solution $\rho(x, t)$ to model (1) with $\alpha=\beta=0.9$.

$$
\begin{aligned}
& N_{1}[\Theta(x, t ; q), \Lambda(x, t ; q)] \\
& ={ }^{c} D_{0, t}^{\alpha} \Theta(x, t ; q)+\frac{c_{0}^{2}}{b_{0}} D_{0, x}^{\beta} \Lambda(x, t ; q), \\
& N_{2}[\Lambda(x, t ; q), \Theta(x, t ; q)] \\
& ={ }^{c} D_{0, t}^{\alpha} \Lambda(x, t ; q)+b_{0}{ }^{c} D_{0, x}^{\beta} \Theta(x, t ; q) .
\end{aligned}
$$

Using the abovementioned definitions and Lemma 2, similar to the method of the fractional Cauchy-Riemann equations, we get

$$
v_{0}(x, t)=E_{\beta} x^{\beta},
$$$$
\rho_{0}(x, t)=\sin _{\beta} x^{\beta}
$$$$
v_{1}(x, t)=h \frac{c_{0}^{2}}{b_{0}} \cos _{\beta} x^{\beta} \frac{t^{\alpha}}{\Gamma(1+\alpha)},
$$$$
\rho_{1}(x, t)=h b_{0} E_{\beta} x^{\beta} \frac{t^{\alpha}}{\Gamma(1+\alpha)},
$$$$
v_{2}(x, t)=(1+h) v_{1}+h^{2} c_{0}^{2} E_{\beta} x^{\beta} \frac{t^{2 \alpha}}{\Gamma(1+2 \alpha)},
$$$$
\rho_{2}(x, t)=(1+h) \rho_{1}-h^{2} c_{0}^{2} \sin _{\beta} x^{\beta} \frac{t^{2 \alpha}}{\Gamma(1+2 \alpha)},
$$$$
v_{3}(x, t)=(1+h) v_{2}+\frac{c_{0}^{2}}{b_{0}} I_{0}^{\alpha c} D_{0, t}^{\alpha}(1+h) \rho_{1}
$$$$
-h^{3} \frac{c_{0}^{4}}{b_{0}} \cos _{\beta} x^{\beta} \frac{t^{3 \alpha}}{\Gamma(1+3 \alpha)}
$$

$$
\rho_{3}(x, t)=(1+h) \rho_{2}+b_{0} I_{0}^{\alpha c} D_{0, t}^{\alpha}(1+h) v_{1}
$$

$$
\begin{gathered}
+h^{3} c_{0}^{2} b_{0} E_{\beta} x^{\beta} \frac{t^{3 \alpha}}{\Gamma(1+3 \alpha)}, \\
v_{4}(x, t)=(1+h) v_{3}+(1+h)(\cdots)+h^{4} c_{0}^{4} E_{\beta} x^{\beta} \frac{t^{4 \alpha}}{\Gamma(1+4 \alpha)}, \\
\rho_{4}(x, t)=(1+h) \rho_{3}+(1+h)(\cdots)+h^{4} c_{0}^{4} \sin _{\beta} x^{\beta} \frac{t^{4 \alpha}}{\Gamma(1+4 \alpha)}, \\
\cdots
\end{gathered}
$$

By repeating this procedure for $h=-1$, noting Definitions 6 and 7, we obtain the exact solutions: 


$$
\begin{aligned}
v(x, t)= & \sum_{k=0}^{\infty} v_{2 k}(x, t)+\sum_{k=1}^{\infty} v_{2 k-1}(x, t), \\
= & E_{\beta} x^{\beta}\left(1+\frac{\left(c_{0} t^{\alpha}\right)^{2}}{\Gamma(1+2 \alpha)}+\frac{\left(c_{0} t^{\alpha}\right)^{4}}{\Gamma(1+4 \alpha)}+\cdots\right) \\
& -\frac{c_{0}}{b_{0}} \cos _{\beta} x^{\beta}\left(\frac{c_{0} t^{\alpha}}{\Gamma(1+\alpha)}-\frac{\left(c_{0} t^{\alpha}\right)^{3}}{\Gamma(1+3 \alpha)}+\cdots\right), \\
= & E_{\beta} x^{\beta} \cosh _{\alpha}\left(c_{0} t^{\alpha}\right)-\frac{c_{0}}{b_{0}} \cos _{\beta} x^{\beta} \sin _{\alpha}\left(c_{0} t^{\alpha}\right) \\
\rho(x, t)= & \sum_{k=0}^{\infty} \rho_{2 k}(x, t)+\sum_{k=1}^{\infty} \rho_{2 k-1}(x, t), \\
= & \sin _{\beta} x^{\beta}\left(1-\frac{\left(c_{0} t^{\alpha}\right)^{2}}{\Gamma(1+2 \alpha)}+\frac{\left(c_{0} t^{\alpha}\right)^{4}}{\Gamma(1+4 \alpha)}+\cdots\right) \\
& -\frac{b_{0}}{c_{0}} E_{\beta} x^{\beta}\left(\frac{c_{0} t^{\alpha}}{\Gamma(1+\alpha)}+\frac{\left(c_{0} t^{\alpha}\right)^{3}}{\Gamma(1+3 \alpha)}+\cdots\right), \\
= & \sin _{\beta} x^{\beta} \cos _{\alpha}\left(c_{0} t^{\alpha}\right)-\frac{b_{0}}{c_{0}} E_{\beta} x^{\beta} \sinh _{\alpha}\left(c_{0} t^{\alpha}\right) . \\
&
\end{aligned}
$$

More interestingly, when $\alpha=1$ and $\beta=1$,

$$
\begin{aligned}
& v(x, t)=e^{x} \cosh \left(c_{0} t\right)-\frac{c_{0}}{b_{0}} \cos x \sin \left(c_{0} t\right), \\
& \rho(x, t)=\sin x \cos \left(c_{0} t\right)-\frac{b_{0}}{c_{0}} e^{x} \sinh \left(c_{0} t\right),
\end{aligned}
$$

are the exact solutions of the following equations:

$$
\left\{\begin{array}{l}
v_{t}(x, t)+\frac{c_{0}^{2}}{b_{0}} \rho_{x}(x, t)=0, \\
\rho_{t}(x, t)+b_{0} v_{x}(x, t)=0,
\end{array}\right.
$$

with initial conditions $v(x, 0)=e^{x}, \rho(x, 0)=\sin x$. In Figures 13-16, the absolute errors of exact solutions and approximate solutions for $v(x, t)$ and $\rho(x, t)$ with differential fractional order $\alpha$ and $\beta$ are shown, which indicates the high efficiency and precision of this method. In the process of compiling the program, we calculate $\sinh _{\alpha, \beta}(x)$ and $\cosh _{\alpha, \beta}(x)$ by the formulas $\sinh _{\alpha, \beta}(Z)=\left(\left(E_{\alpha, \beta}(Z)-\right.\right.$ $\left.\left.E_{\alpha, \beta}(-Z)\right) / 2\right)$ and $\cosh _{\alpha, \beta}(Z)=\left(\left(E_{\alpha, \beta}(Z)+E_{\alpha, \beta}(-Z)\right) / 2\right)$, respectively.

\subsection{Two-Dimensional Space Partial Differential Equation} with a Time-Fractional-Order. In this section, we use the HAM to solve the two-dimensional space partial differential equation with a time-fractional-order of the following form:

$$
{ }^{c} D_{0, t}^{\alpha} u(x, y, t)=\frac{1}{\varphi^{2}+\psi^{2}} \Delta u(x, y, t),
$$

with initial conditions

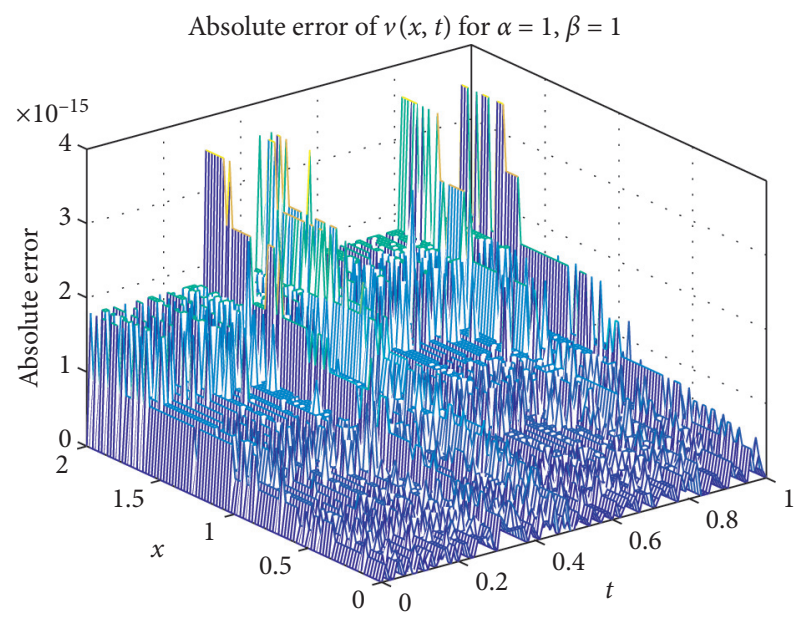

FIGURE 13: Absolute error of the exact solution and approximate solution $v(x, t)$ to fractional acoustic wave equation (2) with $\alpha=\beta=1$.

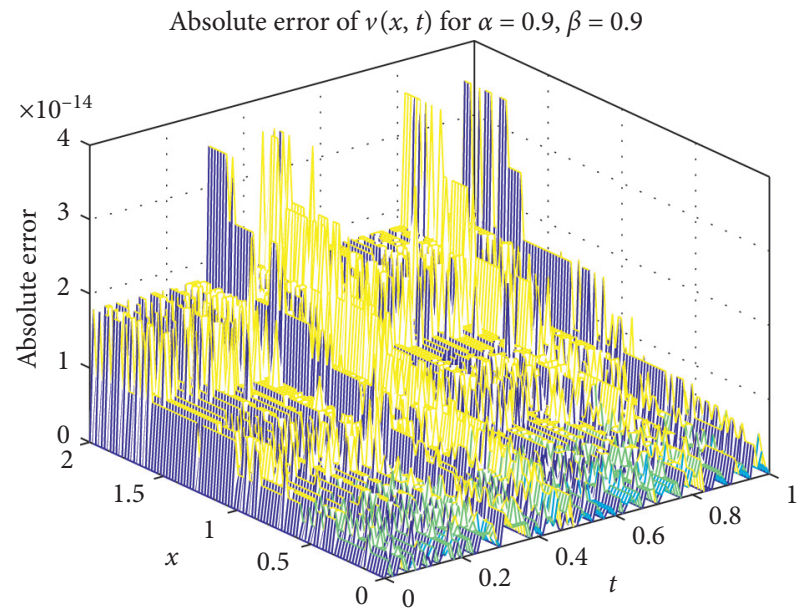

Figure 14: Absolute error of the exact solution and approximate solution $v(x, t)$ to fractional acoustic wave equation (2) with $\alpha=\beta=0.9$.

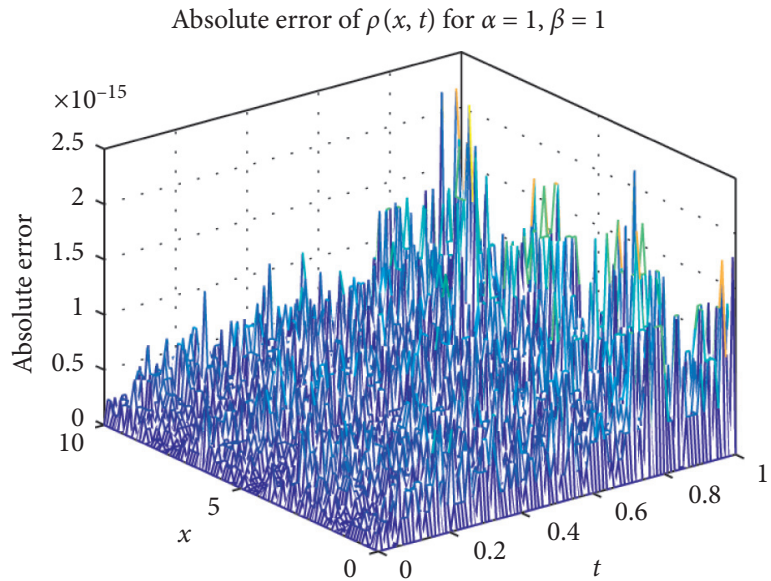

FIGURE 15: Absolute error of the exact solution and approximate solution $\rho(x, t)$ to fractional acoustic wave equation (2) with $\alpha=\beta=1$. 


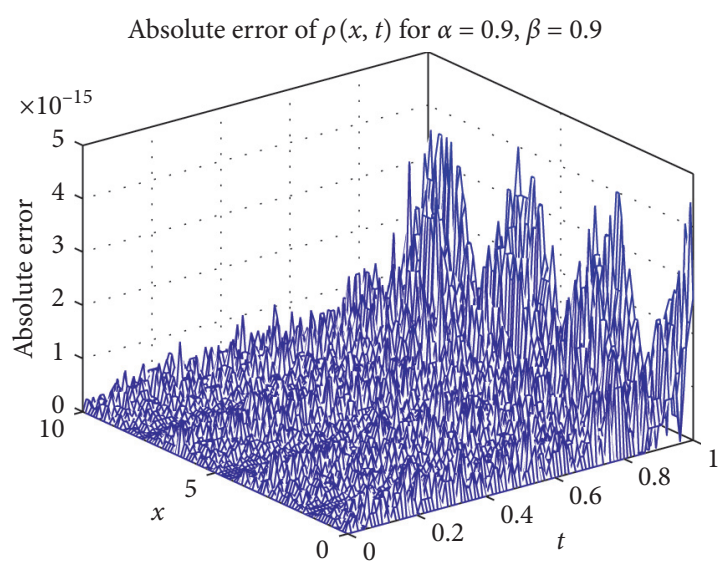

Figure 16: Absolute error of the exact solution and approximate solution $\rho(x, t)$ to fractional acoustic wave equations with (2) $\alpha=\beta=0.9$.

$$
u(x, y, 0)=\cos \left(\frac{2 \pi}{\varphi} x\right) \cos \left(\frac{2 \pi}{\psi} y\right), \quad u_{t}(x, y, 0)=0
$$

and boundary conditions

$$
u(0, y, t)=u(\varphi, y, t)=u(x, 0, t)=u(x, \psi, t)=1,
$$

where $1<\alpha \leq 2$. First, we choose the linear fractional order operator

$$
L[\Theta(x, y, t ; q)]={ }^{c} D_{0, t}^{\alpha} \Theta(x, y, t ; q) .
$$

Secondly, we define the linear operator as

$$
N[\Theta(x, y, t ; q)]={ }^{c} D_{0, t}^{\alpha} \Theta(x, y, t ; q)-\frac{\Theta_{x x}+\Theta_{y y}}{\varphi^{2}+\psi^{2}} .
$$

Using the abovementioned definitions and with assumption $H(x, t)=1$, we construct the zeroth-order deformation equation(ZDE):

$$
(1-q) L\left[\Theta(x, y, t ; q)-u_{0}(x, y, t)\right]=q h N[\Theta(x, y, t ; q)] .
$$

Obviously, when $q=0$ and $q=1$, it holds

$$
\begin{aligned}
\Theta(x, y, t ; 0) & =u_{0}(x, y, t)=u(x, y, 0) \\
& =\cos \left(\frac{2 \pi}{\varphi} x\right) \cos \left(\frac{2 \pi}{\psi} y\right), \\
\varphi(x, y, t ; 1) & =u(x, y, t) .
\end{aligned}
$$

Thirdly, differentiating the ZDE $m$ times with respect to $q$, then setting $q=0$, and dividing it by $m$ !, we get the $m$ thorder deformation equation:

$$
L\left[u_{m}-\chi_{m} u_{m-1}\right]=h N\left[R_{m}\left(\overrightarrow{u_{m-1}}\right)\right],
$$

where

$$
\begin{aligned}
N\left[R_{m}\left(\overrightarrow{u_{m-1}}\right)\right] & ={ }^{c} D_{0, t}^{\alpha} u_{m-1}-\frac{u_{m-1, x x}+u_{m-1, y y}}{\varphi^{2}+\psi^{2}}, \\
u_{m} & =\left.\frac{1}{m !} \frac{\partial^{m} \varphi(x, y, t ; q)}{\partial q^{m}}\right|_{q=0}, \\
\chi_{m} & = \begin{cases}0, & m=1, \\
1, & m>1 .\end{cases}
\end{aligned}
$$

Finally, operating the operator $L^{-1}$ on both the sides of equation (67), we have

$$
u_{m}=\chi_{m} u_{m-1}+h L^{-1} N\left[R_{m}\left(\overrightarrow{u_{m-1}}\right)\right] .
$$

Calculating one by one, we get

$$
\begin{aligned}
& u_{0}=\cos \left(\frac{2 \pi}{\varphi} x\right) \cos \left(\frac{2 \pi}{\psi} y\right), \\
& u_{1}=h\left(\frac{2 \pi}{\varphi \psi}\right)^{2} \cos \left(\frac{2 \pi}{\varphi} x\right) \cos \left(\frac{2 \pi}{\psi} y\right) \frac{t^{\alpha}}{\Gamma(1+\alpha)},
\end{aligned}
$$$$
u_{2}=(1+h) u_{1}+h^{2}\left(\frac{2 \pi}{\varphi \psi}\right)^{4} \cos \left(\frac{2 \pi}{\varphi} x\right) \cos \left(\frac{2 \pi}{\psi} y\right) \frac{t^{2 \alpha}}{\Gamma(1+2 \alpha)},
$$$$
u_{3}=(1+h) u_{2}-\frac{h}{\varphi^{2}+\psi^{2}} I_{0}^{\alpha}\left((1+h) u_{1, x x}+(1+h) u_{1, y y}\right)
$$$$
+h^{3}\left(\frac{2 \pi}{a b}\right)^{6} \cos \left(\frac{2 \pi}{\varphi} x\right) \cos \left(\frac{2 \pi}{\psi} y\right) \frac{t^{3 \alpha}}{\Gamma(1+3 \alpha)}
$$

$u_{4}=(1+h) u_{3}-\frac{h\left(I_{0}^{\alpha}\left((1+h) u_{2, x x}+(1+h) u_{2, y y}\right)\right)}{\varphi^{2}+\psi^{2}}$

$$
\begin{aligned}
& +\frac{h^{2}}{\left(\varphi^{2}+\psi^{2}\right)^{2}} I_{0}^{\alpha}\left(\left(I_{0}^{\alpha}(1+h) u_{1, x x}+(1+h) u_{1, y y}\right)\right)_{x x} \\
& +\left(I_{0}^{\alpha}\left((1+h) u_{1, x x}+(1+h) u_{1, y y}\right)_{y y}\right) \\
& +h^{4}\left(\frac{2 \pi}{\varphi \psi}\right)^{8} \cos \left(\frac{2 \pi}{\varphi} x\right) \cos \left(\frac{2 \pi}{\psi} y\right) \frac{t^{4 \alpha}}{\Gamma(1+4 \alpha)}
\end{aligned}
$$

By repeating this procedure for $h=-1$, we get the exact solution, 


$$
\begin{aligned}
u(x, y, t)= & \sum_{k=0}^{\infty} u_{k}=\sum_{k=0}^{\infty} u_{2 k}+\sum_{k=1}^{\infty} u_{2 k-1} \\
= & \cos \left(\frac{2 \pi}{\varphi} x\right) \cos \left(\frac{2 \pi}{\psi} y\right) \cosh _{\alpha}\left(\left(\frac{2 \pi}{\varphi \psi}\right)^{2} t^{\alpha}\right) \\
& -\cos \left(\frac{2 \pi}{\varphi} x\right) \cos \left(\frac{2 \pi}{\psi} y\right) \sinh _{\alpha}\left(\left(\frac{2 \pi}{\varphi \psi}\right)^{2} t^{\alpha}\right)
\end{aligned}
$$

for example 3. We omit the images of example 3.

\section{Conclusions}

In this paper, using the HAM, we obtained exact solutions for the fractional Cauchy-Riemann equations, fractional acoustic wave equations, and partial differential equation with a time-fractional-order. The absolute errors of the approximate solutions obtained by the HAM show that the approximate solutions are in good agreement with the exact solutions. Fractional differential equations are the generalization of integral ones. In fact, fractional models provide us with an adjustable parameter, which is the order of derivatives.

\section{Data Availability}

Data can be provided by the corresponding author upon reasonable request.

\section{Conflicts of Interest}

The authors declare that they have no conflicts of interest.

\section{Acknowledgments}

This work was partly supported by NSF of China under contract no. 11771265, NSF of Guang-dong Provincial Department of Education under contract nos. 2017KQNCX130, 2018KQNCX156, and 2020KZDZX1147, and Chaozhou Science and Technology Plan Project no. 2019 GY02.

\section{References}

[1] K. B. Oldham and J. Spanier, The Fractional Calculus, Academic Press, New York, NY, USA, 1974.

[2] I. Podlubny, Fractional Differential Equations, Academic Press, New York, NY, USA, 1999.

[3] A. A. Kilbsa, H. M. Srivastava, and J. J. Trujillo, Theory and Applications of Fractional Differential Equations, Elsevier, New York, NY, USA, 2006.

[4] A. I. Saichev and G. M. Zaslavsky, "Fractional kinetic equations: solutions and applications," Chaos: An Interdisciplinary Journal of Nonlinear Science, vol. 7, no. 4, pp. 753-764, 1997.

[5] F. Zeng, F. Liu, C. Li, K. Burrage, I. Turner, and V. Anh, “A Crank--Nicolson ADI spectral method for a two-dimensional Riesz space fractional nonlinear reaction-diffusion equation,"
SIAM Journal on Numerical Analysis, vol. 52, no. 6, pp. 2599-2622, 2014.

[6] R. Metzler and J. Klafter, "Boundary value problems for fractional diffusion equations," Physica A: Statistical Mechanics and its Applications, vol. 278, no. 1-2, pp. 107-125, 2000.

[7] L. Gaul, P. Klein, and S. Kemple, "Damping description involving fractional operators," Mechanical Systems and Signal Processing, vol. 5, no. 2, pp. 81-88, 1991.

[8] Z. Odibat and S. Momani, "Numerical methods for nonlinear partial differential equations of fractional order," Applied Mathematical Modelling, vol. 32, no. 1, pp. 28-39, 2008.

[9] M. M. Meerschaert and C. Tadjeran, "Finite difference approximations for fractional advection-dispersion flow equations," Journal of Computational and Applied Mathematics, vol. 172, no. 1, pp. 65-77, 2004.

[10] I. Podlubny, A. Chechkin, T. Skovranek, Y. Chen, and B. M. Vinagre Jara, "Matrix approach to discrete fractional calculus II: partial fractional differential equations," Journal of Computational Physics, vol. 228, no. 8, pp. 3137-3153, 2009.

[11] A. Elsaid, "Homotopy analysis method for solving a class of fractional partial differential equations," Communications in Nonlinear Science and Numerical Simulation, vol. 16, no. 9, pp. 3655-3664, 2011.

[12] S. Momani and Z. Odibat, "Homotopy perturbation method for nonlinear partial differential equations of fractional order," Physics Letters A, vol. 365, no. 5-6, pp. 345-350, 2007.

[13] S. J. Liao, The proposed homotopy analysis technique for the solution of nonlinear problems, School of Ship and Ocean Engineering, Shanghai Jiao Tong University, Shanghai, China, Ph.D. dissertation, 1992.

[14] S. Liao, "On the homotopy analysis method for nonlinear problems," Applied Mathematics and Computation, vol. 147, no. 2, pp. 499-513, 2004.

[15] L. Song and H. Zhang, "Solving the fractional BBM-Burgers equation using the homotopy analysis method," Chaos, Solitons \& Fractals, vol. 40, no. 4, pp. 1616-1622, 2009.

[16] H. Jafari, A. Golbabai, S. Seifi, and K. Sayevand, "Homotopy analysis method for solving multi-term linear and nonlinear diffusion-wave equations of fractional order," Computers \& Mathematics with Applications, vol. 59, no. 3, pp. 1337-1344, 2010.

[17] X. Hang, J. L. Shi, and C. Y. Xiang, "Analysis of nonlinear fractional partial differential equations with the homotopy analysis method," Communications in Nonlinear Science and Numerical Simulation, vol. 14, pp. 1152-1156, 2009.

[18] Z. Odibat, S. Momani, and H. Xu, "A reliable algorithm of homotopy analysis method for solving nonlinear fractional differential equations," Applied Mathematical Modelling, vol. 34, no. 3, pp. 594-600, 2010.

[19] L. Song and H. Zhang, "Application of homotopy analysis method to fractional KdV-Burgers-Kuramoto equation," Physics Letters A, vol. 367, no. 1-2, pp. 88-94, 2007.

[20] H. Xu and J. Cang, "Analysis of a time fractional wave-like equation with the homotopy analysis method," Physics Letters A, vol. 372, no. 8, pp. 1250-1255, 2008.

[21] I. Hashim, O. Abdulaziz, and S. Momani, "Homotopy analysis method for fractional IVPs," Communications in Nonlinear Science and Numerical Simulation, vol. 14, no. 3, pp. 674-684, 2009.

[22] M. Dehghan, J. Manafian, and A. Saadatmandi, "Solving nonlinear fractional partial differential equations using the homotopy analysis method," Numerical Methods for Partial Differential Equations, vol. 26, no. 2, pp. 448-479, 2009. 
[23] S. Abbasbandy, M. S. Hashemi, and I. Hashim, "On convergence of homotopy analysis method and its application to fractional integro-differential equations," Quaestiones Mathematicae, vol. 36, no. 1, pp. 93-105, 2013.

[24] V. F. Morales-Delgado, J. F. Gómez-Aguilar, H. YépezMartínez et al., "Laplace homotopy analysis method for solving linear partial differential equations using a fractional derivative with and without kernel singular," Advances in Difference Equations, vol. 2016, no. 1, p. 164, 2016.

[25] Z. Odibat, "On the optimal selection of the linear operator and the initial approximation in the application of the homotopy analysis method to nonlinear fractional differential equations," Applied Numerical Mathematics, vol. 137, pp. 203-212, 2019.

[26] H. Ye'pez-Martínez and J. F. Gómez-Aguilar, "A new modified definition of Caputo Fabrizio fractional-order derivative and their applications to the multi step homotopy analysis method," Journal of Computational and Applied Mathematics, vol. 346, pp. 247-260, 2019.

[27] Z. Odibat and D. Baleanu, "A linearization-based approach of homotopy analysis method for non-linear time-fractional parabolic PDEs," Mathematical Methods in the Applied Sciences, vol. 42, no. 18, pp. 7222-7232, 2019.

[28] K. M. Saad, E. H. F. AL-Shareef, A. K. Alomari, D. Baleanu, and J. F. Gómez-Aguilar, "On exact solutions for time-fractional Korteweg-de Vries and Korteweg-de Vries-Burger's equations using homotopy analysis transform method," Chinese Journal of Physics, vol. 63, pp. 149-162, 2020.

[29] S. G. Krantz, "The Cauchy-Riemann equations," in Geometric Function Theory, Cornerstones, S. G. Krantz, Ed., Birkhäuser, Boston, MA, USA, 2006.

[30] C. Cerjan, D. Kosloff, R. Kosloff, and M. Reshef, "A nonreflecting boundary condition for discrete acoustic and elastic wave equations," Geophysics, vol. 50, no. 4, pp. 705-708, 1985.

[31] I. Podlubny, "Mittag-Leffler function matlab central," http:// www.mathworks.com/matlabcentral/fileexchange/8738-mittagleffler-function, 2012. 